\title{
Physiological changes in winter wheat genotypes in response to the Zymoseptoria tritici infection
}

\author{
G. MIHAILOVA*, Z. STOYANOVA*, R. RODEVA*, B. BANKINA**, G. BIMŠTEINE*, \\ and K. GEORGIEVA*,+
}

Institute of Plant Physiology and Genetics, Bulgarian Academy of Sciences, Acad. G. Bonchev Street, Bl. 21, 1113

Sofia, Bulgaria*

Faculty of Agriculture, Latvia University of Agriculture, Liela Street 2, LV-3001 Jelgava, Latvia**

\begin{abstract}
Some physiological aspects of interactions between wheat and Zymoseptoria tritici were studied by measuring changes in some stress markers, photosynthetic activity, and the extent of thermal energy dissipation. Four winter wheat genotypes varying in their level of resistance, determined on the basis of necrotic leaf area and presence of pycnidia, were inoculated at a seedling stage and measurements were performed at $0,2,17$, and $23 \mathrm{~d}$ after the inoculation. Physiological activity of wheat genotypes showed that keeping the membrane integrity and the extent of oxidative stress is of significant importance for pathogen resistance. It could be suggested that the changes in electrolyte leakage, malondialdehyde content, and values of quantum yield of PSII electron transport and nonphotochemical fluorescence quenching can be used to screen the degree of tolerance to Septoria tritici blotch in different wheat genotypes.
\end{abstract}

Additional key words: chlorophyll fluorescence; fungal infection; photosynthesis; Triticum aestivum.

\section{Introduction}

Wheat (Triticum aestivum L.) is the most widely distributed crop with the highest production in European agriculture. Of the various pathogens, Zymoseptoria tritici (Desm.) Quaedvlieg and Crous (formerly known as Septoria tritici Rob. ex Desm., anamorph, and Mycosphaerella graminicola (Fückel) J. Schröter in Cohn, teleomorph), is one of the most economically important wheat-infecting fungi in Europe and worldwide (Dean et al. 2012, Fones and Gurr 2015), which causes a devastating foliar disease Septoria tritici blotch (STB). This polycyclic pathogen occurs early in the growing season and can accomplish up to six cycles on the wheat crop (Fones and Gurr 2015). Typical symptoms of STB appear 14-21 d after infection and consist of irregular gray-brown flecking, where light to dark brown pycnidia develop. These necrotic lesions reduce photosynthetic capacity of leaves. Damage of infected leaf tissue can induce complex changes in the plant's carbon metabolism and assimilate partitioning. The disease causes large losses (up to 50\%) in wheat yield usually related to the losses of green area (Eyal et al. 1987).
Zymoseptoria tritici is a hemibiotrophic pathogen with biphasic lifestyle (Rudd et al. 2015). The first infection phase, frequently referred to as biotrophic, undergoes asymptomatically and is unusually prolonged up to $28 \mathrm{~d}$ depending on the specific combination between wheat genotype and fungal isolate (Lee et al. 2014). After conidial germination and fungal invasion through the stomata, the hyphae grow very slowly in the apoplastic space between mesophyll cells (Kema et al. 1996). This phase is also characterized by the absence, or very weak defense response (Rudd et al. 2015). The second infection phase, known as necrotrophic, is symptomatic (Kema et al. 1996, Steinberg 2015). It is typified by a rapid induction of cell death and loss of membrane permeability. The necrotrophic feeding phase is required for build-up of fungal biomass and asexual sporulation (Kema et al. 1996, Dean et al. 2012). At the disease transition phase all processes are activated in the plants but the energy is diverted away from any further photosynthesis in the infected tissue (Rudd et al. 2015). The majority of plant cell wall-attacking enzymes are strongly expressed at the necrotrophic phase (Brunner et al. 2013). In contrast,

\footnotetext{
$\overline{\text { Received }} 26$ June 2018, accepted 28 November 2018.

${ }^{+}$Corresponding author; e-mail: georgieva.katya.m@gmail.com, katya@bio21.bas.bg

Abbreviations: $1-\left(\mathrm{F}_{\mathrm{v}}{ }^{\prime} / \mathrm{F}_{\mathrm{m}}{ }^{\prime}\right)$ - relative proportion of the energy absorbed and dissipated as heat in the PSII antennae; Chl - chlorophyll; dpi - days post inoculation; FM - fresh mass; $\mathrm{F}_{\mathrm{v}} / \mathrm{F}_{\mathrm{m}}$ - maximum quantum efficiency of PSII; $g_{\mathrm{s}}$ - stomatal conductance; MDA malondialdehyde; NPQ - nonphotochemical fluorescence quenching; $\mathrm{q}_{\mathrm{P}}$ - photochemical quenching; RFd - ratio of Chl fluorescence decrease to the steady state $\mathrm{Chl}$ fluorescence; STB - Septoria tritici blotch; $\Delta \mathrm{A}_{820}$ - far-red light-induced P700 oxidation; $\Phi_{\mathrm{PSII}}-$ quantum yield of PSII electron transport in the light-adapted state.

Acknowledgements: The present investigation was carried out within the framework of bilateral research project between Bulgarian and Latvian Academy of Sciences.
} 
transcripts encoding photosynthesis processes are strongly downregulated during the rapid fungal colonization (Rudd et al. 2015).

Lately, much attention has been focused on different aspects of the compatible and incompatible interactions between fungus and its host including transcriptome and metabolite profiling (Brunner et al. 2013, Lee et al. 2014, Rudd et al. 2015, Steinberg 2015, Palma-Guerrero et al. 2016, Orton et al. 2017). The information about photochemical processes in the wheat plant affected by Z. tritici seems to be insufficient (Robert et al. 2006).

This investigation was undertaken to detect alterations in the photosynthetic activity due to STB in four winter wheat genotypes varying in their level of resistance, determined on the basis of necrotic leaf area and presence of pycnidia. The consequence of $Z$. tritici inoculation was evaluated by measuring the changes in electrolyte leakage, malondialdehyde (MDA), proline, chlorophyll (Chl) and carotenoid (Car) contents, photochemical activity of PSI and PSII, stomatal conductance $\left(g_{\mathrm{s}}\right)$, and the extent of thermal energy dissipation. The time points of the assessments were chosen to estimate the effect of inoculation before the onset of symptoms, at the switch to the necrotrophic growth and after the diseased tissue became necrotic.

\section{Materials and methods}

Plant material and treatments: Four winter wheat genotypes (Triticum aestivum L.) were included in the study. They showed a different resistance to Z. tritici: a highly susceptible (Bulgarian cv. Enola), a highly resistant (Swiss cv. Arina), and two moderately resistant (Bulgarian cv. Milena and Latvian advanced line L-325 12-16, further referred as L325). Arina is one of the most STB-resistant European wheat cultivars (Brown et al. 2001). It is a good source of partial resistance to STB in the field (resistant quantitative trait loci). Besides, two major genetic loci for resistance to Z. tritici (Stb6 and Stb15) have been identified and mapped in cv. Arina (Arraiano et al. 2007).

A greenhouse pot experiment was conducted in a complete randomized block design with five replications at approximately $20 / 12^{\circ} \mathrm{C}$ day/night temperature. Metal terrines $(35 \times 35 \times 8 \mathrm{~cm})$ filled with the same mass parts of the fertilizer and soil mixture were used. The chemical content of fertilizer was: $180 \mathrm{mg}$ (ammonium nitrate) $\mathrm{kg}^{-1}$ (soil), $65 \mathrm{mg}$ (triple superphosphate) $\mathrm{kg}^{-1}$ (soil), and $42 \mathrm{mg}$ (potassium chloride) $\mathrm{kg}^{-1}$ (soil). Fifty good, clean, and healthy seeds were sown in each tray. Inoculation was carried out 3 weeks after sowing at a growth stage 12 (Zadoks et al. 1974), when the second leaf was fully expanded. Seedlings in each tray were thinned to the 36 the most uniform plants and then inoculated.

A single pycnidiospore isolate of Z. tritici (Zt1-17) obtained from a naturally infected winter wheat (cv. Enola) was used for all plants. This isolate was multiplied on potato-dextrose agar in a growth chamber at $18^{\circ} \mathrm{C}$. Conidia were collected from 7-d-old cultures in sterile distilled water. Before inoculation, suspension concentration was adjusted to about $10^{6}$ conidia $\mathrm{ml}^{-1}$ using a haemocytometer
(Thoma, Fein-Optik, Germany) and 0.04\% Tween 20 was added for uniform dispersion of conidia. The plants in every tray were evenly sprinkled with a hand sprayer applying $50 \mathrm{ml}$ of the suspension. Five trays (replicates) were inoculated for each cultivar. Noninoculated control plants received the same treatments without the fungal conidia using just Tween 20 water solution. Immediately after the spraying, the trays were put at $100 \%$ relative humidity and $20^{\circ} \mathrm{C}$ for $96 \mathrm{~h}$. New leaves appearing after inoculation were removed at 5-d intervals.

Development of STB symptoms (chlorotic and necrotic) was regularly observed. Necrotic symptoms included sporulating and nonsporulating necrosis. The incubation and latent periods were determined as the number of days from inoculation to the appearance of the first visible symptoms and the first pycnidia, respectively. Disease severity was measured as percentage of the leaf area showing symptoms. The amount of pycnidia was determined according to the scale of Rosielle (1972).

All measurements were performed on the second leaf. The samples for the first assessment were collected at the day of inoculation just before spraying. Second sampling date was $2 \mathrm{~d}$ after inoculation (dpi). The third assessment was performed when the first visible necrotic symptoms occurred (17 dpi) and the last one at full manifestation of symptoms on the most susceptible genotype (23 dpi).

The relative water content (RWC) of leaves was determined gravimetrically by weighing before and after ovendrying at $80^{\circ} \mathrm{C}$ to a constant mass and expressed as the percentage of water content in dehydrated tissue compared to water-saturated tissues, using the equation: RWC [\%] = $\left(m_{\text {fresh }}-m_{\text {dry }}\right) \times 100 /\left(m_{\text {saturated }}-m_{\text {dry }}\right)$, where $m_{\text {fresh }}$ is the fresh mass, $\mathrm{m}_{\text {dry }}$ is the dry mass, and $\mathrm{m}_{\text {saturated }}$ is the saturated mass. $m_{\text {saturated }}$ was measured on leaf discs maintained 12 $16 \mathrm{~h}$ at $4^{\circ} \mathrm{C}$ in the dark floating on water.

Electrolyte leakage from leaf tissue was measured with a conductivity meter (EC 215, Hanna Instruments, USA) after 24-h incubation of leaf disks in double-distilled water. The maximum leakage of the tissue was determined by boiling the leaves for $15 \mathrm{~min}$ at $100^{\circ} \mathrm{C}$. The results are expressed as percentage of maximum leakage.

Pigment determination: Chlorophyll (Chl) $a, \mathrm{Chl} b$, and total Car were extracted from leaf disks with $80 \%$ acetone. The pigment content was determined spectrophotometrically (Multiskan Spectrum, Thermo Scientific, USA) according to Lichtenthaler (1987) and the data were calculated on a fresh mass basis.

MDA content was determined as described by Esterbauer and Cheeseman (1990). Leaf material (250 mg) was homogenized at $4^{\circ} \mathrm{C}$ in $2.5 \mathrm{ml}$ of $0.1 \%$ trichloracetic acid (TCA), and centrifuged at $15,000 \times g$ for $30 \mathrm{~min}$. The reaction mixture contained $0.5 \mathrm{ml}$ of the supernatant, $0.5 \mathrm{ml}$ of $0.1 \% \mathrm{TCA}$, and $1 \mathrm{ml}$ of $0.5 \%$ thiobarbituric acid in $20 \%(\mathrm{w} / \mathrm{v})$ TCA. This solution was boiled in water bath for $30 \mathrm{~min}$, centrifuged at $4,000 \times \mathrm{g}$ for 10 min, and the absorbance was read spectrophotometrically 
(Multiskan Spectrum, Thermo Scientific, USA) at 532 and $600 \mathrm{~nm}$ for the determination of MDA. The data were calculated on a fresh mass basis.

Proline content was determined by the method of Bates et al. (1973). Leaves (400 mg) were homogenized in $10 \mathrm{ml}$ of $3 \%$ aqueous sulphosalicylic acid; then the homogenate was centrifuged at $2,000 \times g$ for $5 \mathrm{~min}$. The extract of $2 \mathrm{ml}$ was mixed with $2 \mathrm{ml}$ of acidic-ninhydrine and $2 \mathrm{ml}$ of glacial acetic acid for $1 \mathrm{~h}$ at $100^{\circ} \mathrm{C}$. The reaction mixture was extracted with $4 \mathrm{ml}$ of toluene. The chromophore containing toluene was separated and the absorbance read at $520 \mathrm{~nm}$ (Spekol 11, Carl Zeiss, Jena, Germany), The data were calculated on a fresh mass basis.

Chlorophyll fluorescence was measured with a portable fluorometer PAM-2500 (Heinz Walz GmbH, Effeltrich, Germany). The maximal PSII quantum efficiency was calculated as $\mathrm{F}_{\mathrm{v}} / \mathrm{F}_{\mathrm{m}}$ immediately after dark adaptation. For the measurements, actinic light of $400 \mu \mathrm{mol}$ (photon) $\mathrm{m}^{-2} \mathrm{~s}^{-1}$ was used. The actual efficiency of PSII electron transport during illumination was estimated at steady state as $\Phi_{\mathrm{PSII}}=\left(\mathrm{F}_{\mathrm{m}}{ }^{\prime}-\mathrm{F}_{\mathrm{s}}\right) / \mathrm{F}_{\mathrm{m}}{ }^{\prime}($ Genty et al. 1989), where $\mathrm{F}_{\mathrm{m}}{ }^{\prime}$ is the maximum fluorescence and $\mathrm{F}_{\mathrm{s}}$ is the steadystate fluorescence under illumination. The photochemical quenching, $\mathrm{q}_{\mathrm{P}}$, was estimated as $\mathrm{q}_{\mathrm{P}}=\left(\mathrm{F}_{\mathrm{m}}{ }^{\prime}-\mathrm{F}_{\mathrm{s}}\right) /\left(\mathrm{F}_{\mathrm{m}}{ }^{\prime}-\mathrm{F}_{\mathrm{o}}{ }^{\prime}\right)$ according to Schreiber et al. (1986) as formulated by van Kooten and Snel (1990). Stern-Volmer type nonphotochemical fluorescence quenching (NPQ) (Bilger and Björkman 1990) was estimated as NPQ $=F_{m} / F_{m}{ }^{\prime}-1$ and the ratio of $\mathrm{Chl}$ fluorescence decrease to the steady-state Chl fluorescence as $\mathrm{RFd}=\left(\mathrm{F}_{\mathrm{m}}{ }^{\prime}-\mathrm{F}_{\mathrm{s}}\right) / \mathrm{F}_{\mathrm{s}}$ (Lichtenthaler et al. 2007). The relative proportion of the energy absorbed and dissipated as heat in the PSII antennae was estimated by $1-\left(\mathrm{F}_{\mathrm{v}}{ }^{\prime} / \mathrm{F}_{\mathrm{m}}{ }^{\prime}\right)$ (Demmig-Adams et al. 1996).

Reoxidation of P700: The redox state of P700 was monitored in vivo as $810 / 860 \mathrm{~nm}$ absorption changes $\left(\Delta \mathrm{A}_{820}\right)$ using a Walz ED 700DW-E emitter/detector connected to a PAM 101E main control unit (Walz, Effeltrich, Germany). P700 was oxidized by far-red (FR) light from a photodiode (FR-102, Walz, Effeltrich, Germany). Intensity of FR light was $13.4 \mathrm{~W} \mathrm{~m}^{-2}$. The maximum absorption of the $\mathrm{Chl}$ molecule in the reaction center of PSI, $\mathrm{P} 700^{+}$, is around $820 \mathrm{~nm}$

The stomatal conductance $\left(\boldsymbol{g}_{\mathrm{s}}\right)$ was measured on the adaxial side of the leaves using a leaf porometer $S C-1$ (Decagon Devices, Inc., Pullman, WA, USA) equipped with desiccant chamber. Before measurements the sensor head was calibrated to ensure accurate conductance reading.

Statistical analysis: All measurements of physiological parameters were conducted in six replications. For Chl fluorescence measurements at each time point, two leaves from each cultivar in each replicate were collected and pooled as one sample. Control (noninoculated) and inoculated samples were statistically compared by the Fisher's least significant difference test at $P \leq 0.05$ following analysis of variance (ANOVA). A statistical software package (StatGraphics Plus, version 5.1 for Windows, USA) was used.

The Pearson's correlation coefficient $(r)$ was used to measure the strength of a linear association between two variables. It was calculated in Microsoft Excel. The formulas return a value between -1 and 1 , where 1 indicates a strong positive relationship and -1 indicates a strong negative relationship. A result of zero indicates no relationship at all.

\section{Results}

STB symptom development: The inoculated leaves were green during the latent period. The first chlorotic symptoms were observed at $17 \mathrm{dpi}$, most clearly visible in the highly susceptible cv. Enola. Two days later, necrosis with noticeable pycnidia started to develop. Chlorotic tissues were gradually replaced by necrosis (sporulating and nonsporulating areas). At $23 \mathrm{dpi}$, the diseased leaf tissue of the susceptible genotype was completely destroyed and mature pycnidia were present. Then the last measurements were made and clear differences were observed between the wheat genotypes varying in their resistance (Fig. 1S, supplement). On the leaves of highly susceptible cv. Enola, typical symptoms of STB developed displaying as large merging necrotic areas (up to $90 \%$ of leaf surface) containing numerous pycnidia (Fig. 1S $A$ ). Only a few small gray-green elliptic spots without pycnidia were observed on highly resistant cv. Arina (up to 5\%) (Fig. 1SB). On both moderately resistant wheat genotypes (cv. Milena and line L325), a larger necrotic area than that on cv. Arina (up to 20\%) developed mainly on the leaf tip and periphery but none or very few pycnidia were formed (Fig. 1SC). The water-treated controls of all cultivars remained free of disease throughout the experiment.

RWC, electrolyte leakage, MDA, and proline content: The RWC of control wheat plants from all cultivars was not altered during the investigated period. Inoculation of plants with $Z$. tritici slightly declined the RWC (up to $10 \%$ compared to the controls) only at $23 \mathrm{dpi}$ (data not shown).

The effect of $Z$. tritici inoculation on the membrane integrity was assessed by measuring the changes in electrolyte leakage. The values of this parameter were similar in control plants of all wheat cultivars and were not significantly altered in the time course of the experiment (Fig. 1). Inoculation with $Z$. tritici influenced differently the membrane integrity of the studied cultivars. While the extent of electrolyte leakage was not affected in cv. Arina, it gradually rose in the other wheat genotypes reaching the maximum at $23 \mathrm{dpi}$. The strongest increase in electrolyte leakage was found in cv. Enola, where it was 8.6-fold over the control, whereas in line L325 and cv. Milena it increased 3.4- and 3.7-fold compared to the control.

The MDA content, as a measure of lipid peroxidation, significantly increased in all studied genotypes inoculated with Z. tritici as early as the 17 dpi (Fig. 2). Similarly to the electrolyte leakage, the highest MDA values were measured in cv. Enola at 23 dpi. It should be noted that the 


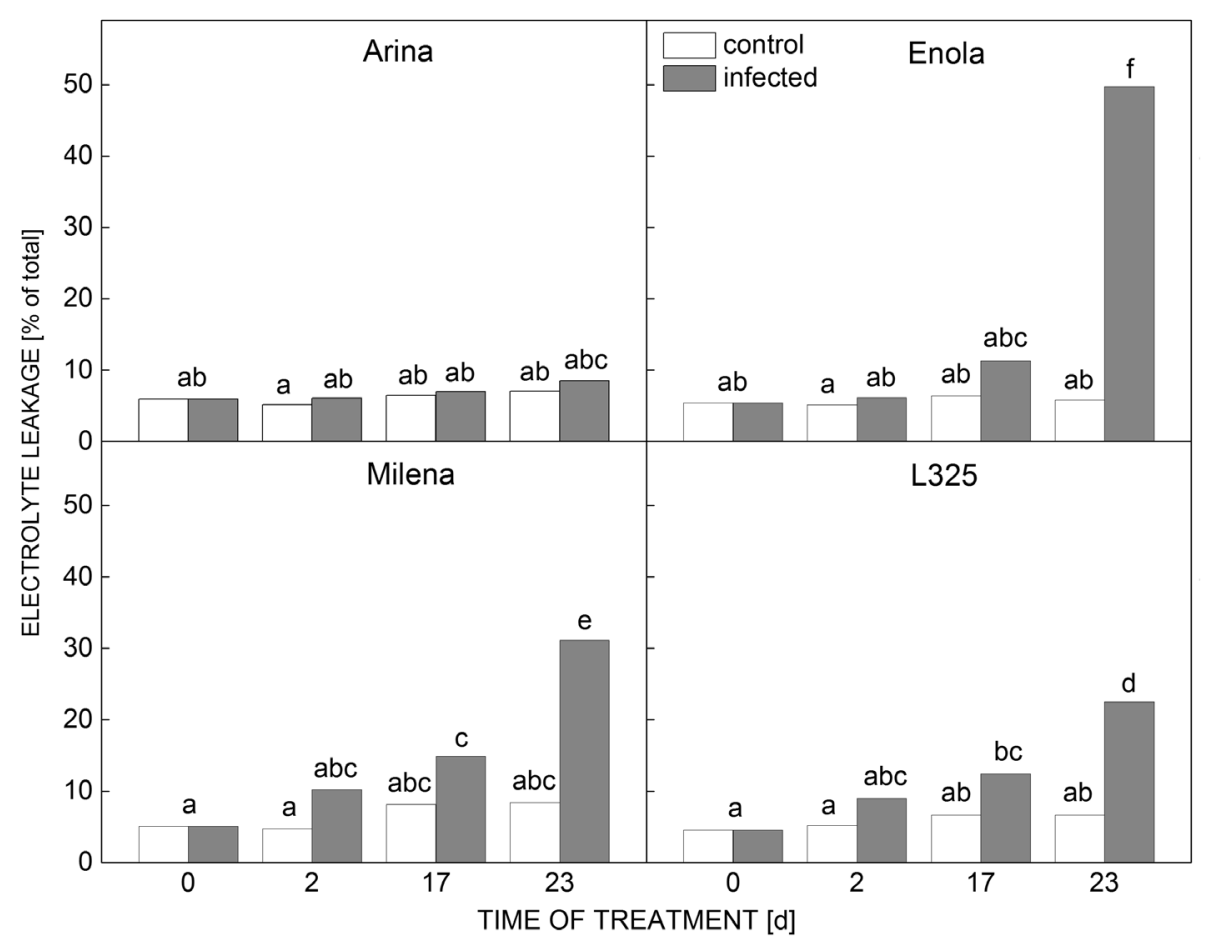

Fig. 1. Changes in the electrolyte leakage caused by inoculation of wheat genotypes Arina (highly resistant), Enola (highly susceptible), Milena, and L325 (moderately resistant) with Zymoseptoria tritici at 0, 2, 17, and 23 days post inoculation. Data represent the mean of $n$ $=6$; the same letters within a graph indicate no significant differences assessed by Fisher's LSD test $(P \leq 0.05)$ after performing $A N O V A$.

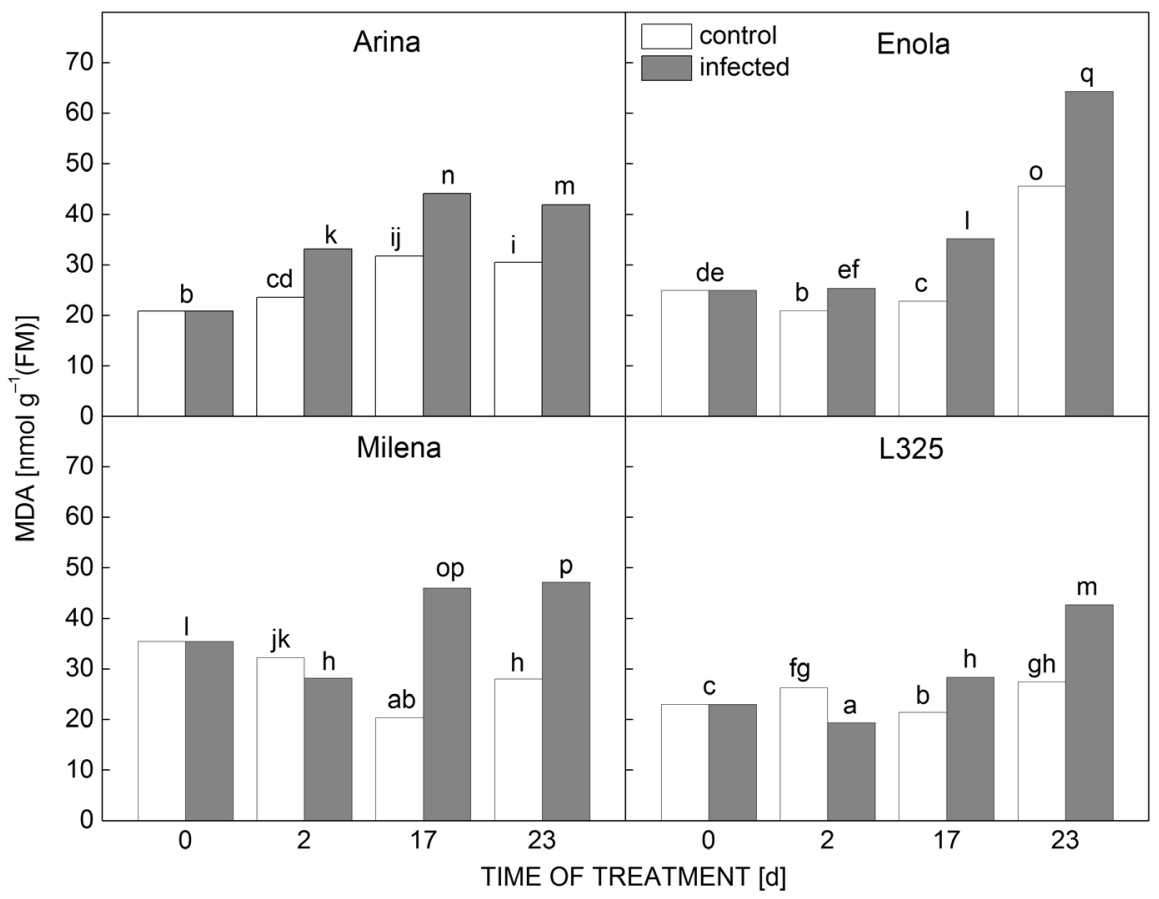

Fig. 2. Changes in the malondialdehyde content caused by inoculation of wheat genotypes Arina (highly resistant), Enola (highly susceptible), Milena, and L325 (moderately resistant) with Zymoseptoria tritici measured at 0, 2, 17, and 23 days post inoculation. Data represent the mean of $n=6$; the same letters within a graph indicate no significant differences assessed by Fisher's LSD test ( $P \leq 0.05)$ after performing $A N O V A$. 


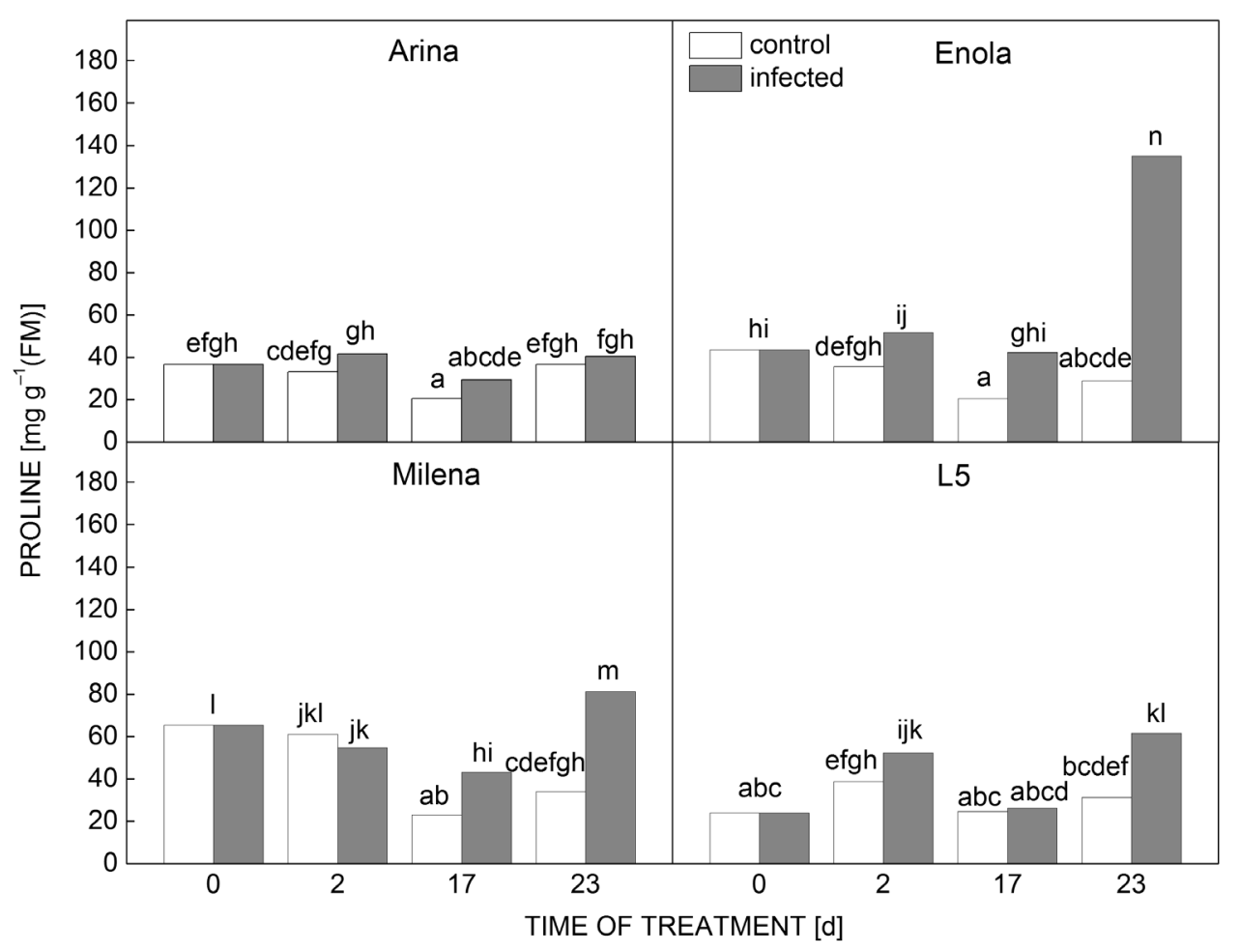

Fig. 3. Effect of inoculation with Zymoseptoria tritici on proline content of wheat genotypes Arina (highly resistant), Enola (highly susceptible), Milena, and L325 (moderately resistant) measured at $0,2,17$, and 23 days post inoculation. Data represent the mean of $n$ =6; the same letters within a graph indicate no significant differences assessed by Fisher's LSD test $(P \leq 0.05)$ after performing ANOVA.

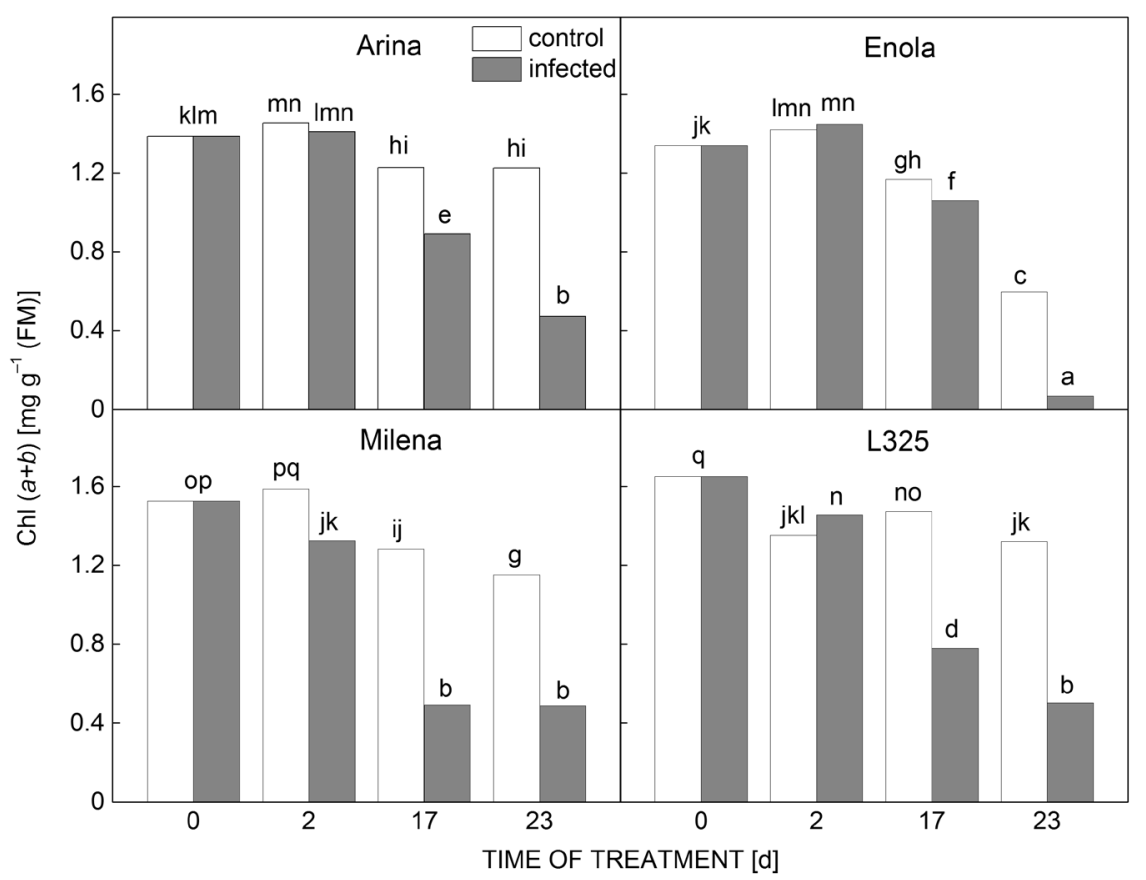

Fig. 4. Changes in the content of chlorophyll $(\mathrm{Chl} a+b)$ caused by inoculation of winter wheat genotypes Arina (highly resistant), Enola (highly susceptible), Milena, and L325 (moderately resistant) with Zymoseptoria tritici at 0, 2, 17, and 23 days post inoculation. Data represent the mean of $n=6$; the same letters within a graph indicate no significant differences assessed by Fisher's LSD test $(P \leq 0.05)$ after performing ANOVA. 
most significant difference in the amount of MDA between 17 and 23 dpi was observed in cv. Enola.

The significant enhancement in electrolyte leakage and MDA content of cv. Enola, Milena, and line L325 at 23 dpi was accompanied with an increase in the proline content (Fig. 3). The latter was doubled compared with the control in cv. Milena and line L325 and it rose 4.7 times in cv. Enola.

Pigment content: The content of Chl $(a+b)$ in control plants decreased throughout the 23-d period, which was different for the wheat genotypes studied (Fig. 4). While the $\mathrm{Chl}$ content declined similarly in the control plants of all genotypes at $17 \mathrm{dpi}$ (by 11-16\%), the strongest reduction in its content was found in cv. Enola at $23 \mathrm{dpi}$ (55\%), followed by cv. Milena and line L325 (24 and 20\%, respectively) and cv. Arina (12\%). Inoculation of wheat plants with $Z$. tritici accelerated the Chl degradation and its content decreased by $60 \%$ in cvs. Arina, Milena, and L325 and about $90 \%$ in cv. Enola compared to the respective controls at $23 \mathrm{dpi}$. The content of Car was also reduced in infected leaves but it was less pronounced than that of Chl (Fig. 2S). The amount declined by $24 \%$ in cv. Arina, about $40 \%$ in cv. Milena and line L325, and $72 \%$ in cv. Enola at 23 dpi.

Photosynthetic activity: Chl fluorescence measurements showed that the infection of wheat plants with $Z$. tritici declined the maximum quantum efficiency of PSII photochemistry (estimated by the ratio $\mathrm{F}_{\mathrm{v}} / \mathrm{F}_{\mathrm{m}}$ ) by $5 \%$ in $\mathrm{cv}$.
Arina, 9 and 14\% in line L325 and cv. Milena, respectively, and about $40 \%$ in cv. Enola compared with the respective controls only at $23 \mathrm{dpi}$ (Fig. $3 \mathrm{~S}$ ). In contrast to $\mathrm{F}_{\mathrm{v}} / \mathrm{F}_{\mathrm{m}}$, the quantum efficiency of electron transport through PSII ( $\left.\Phi_{\text {PSII }}\right)$ declined more throughout the 23-d period (Fig. 5). A significant decrease of $\Phi_{\text {PSII }}$ in control plants from all cultivars was observed after $17 \mathrm{~d}$ but no further changes in this parameter were registered after $23 \mathrm{~d}$. The results showed that inoculation of plants with $Z$. tritici resulted in a further decline in $\Phi_{\mathrm{PSII}}$ by $10 \%$ in cv. Arina and L325, $15 \%$ in cv. Milena, and by $53 \%$ in cv. Enola at $23 \mathrm{dpi}$. It was found that the fungal infection of wheat leaves at 23 dpi reduced the values of $\Phi_{\text {PSII }}$ in cVs. Arina, Milena, and L325 mainly due to decline in $\mathrm{F}_{\mathrm{v}}{ }^{\prime} / \mathrm{F}_{\mathrm{m}}{ }^{\prime}$, whereas it was due to a decrease of both photochemical quenching $\left(\mathrm{q}_{\mathrm{P}}\right)$ and $\mathrm{F}_{\mathrm{v}}{ }^{\prime} / \mathrm{F}_{\mathrm{m}}{ }^{\prime}$ in $\mathrm{cv}$. Enola with major contribution of $\mathrm{q}_{\mathrm{P}}$ (data not shown). Changes in the values of ratio of $\mathrm{Chl}$ fluorescence decrease to the steady state Chl fluorescence, RFd, permit a fast screening of the photosynthetic activity and vitality of plants under stress. The values of RFd were affected by the fungal infection similarly as $\Phi_{\text {PSII }}$ but to higher extent, indicating that this is one of the most sensitive Chl fluorescence parameters (Fig. 4S). The data showed the most significant reduction in RFd values in cv. Enola at 23 dpi $(67 \%)$, followed by cv. Milena (25\%) and cvs. Arina and line L325 (16\%).

Monitoring far-red light-induced P700 oxidation by detecting absorbance changes at around $820 \mathrm{~nm}$ is a convenient way to evaluate electron transport properties through PSI. The alterations in the photochemical activity

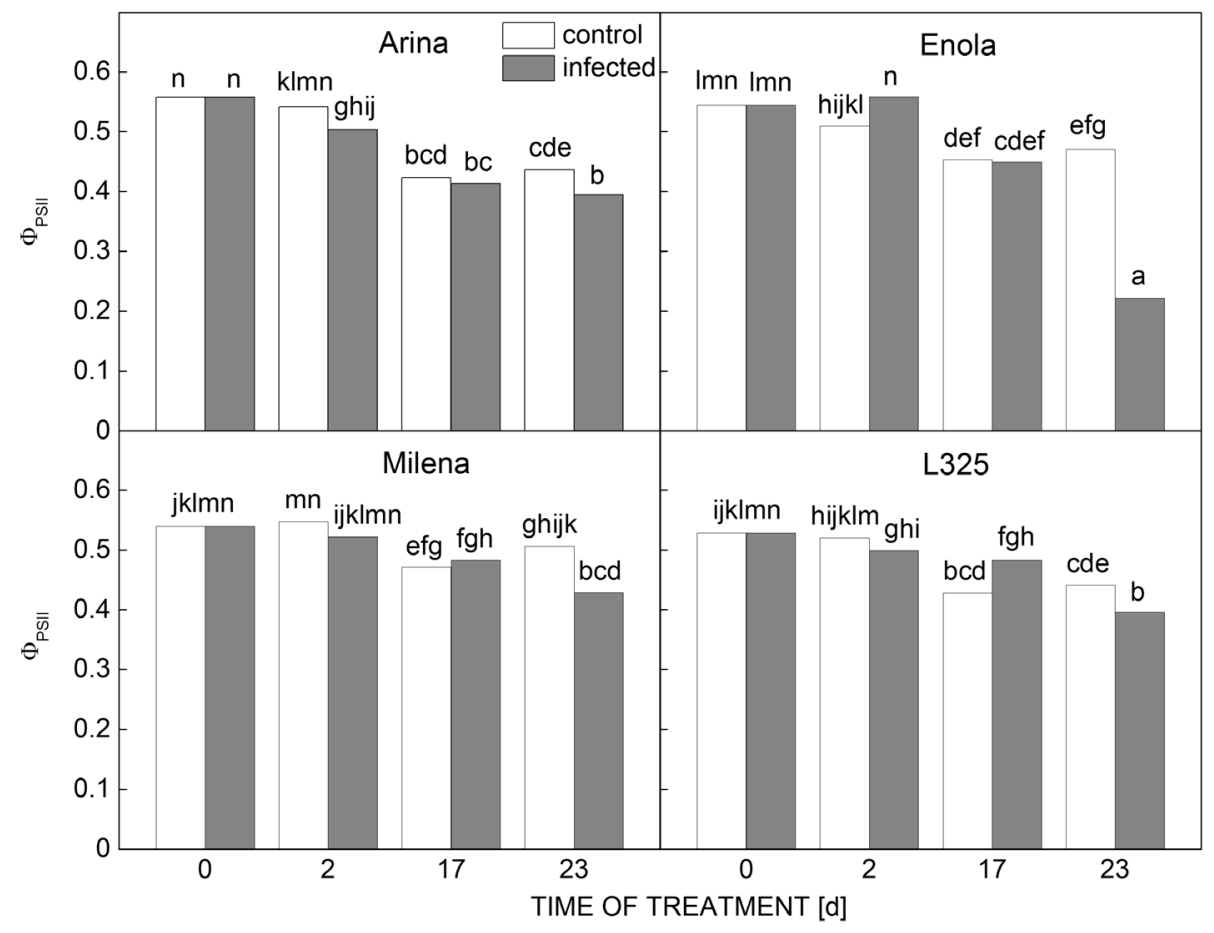

Fig. 5. Quantum yield of PSII electron transport in the light-adapted state, $\Phi_{\text {PSII }}$, of wheat genotypes Arina (highly resistant), Enola (highly susceptible), Milena, and L325 (moderately resistant) inoculated with Zymoseptoria tritici measured at 0, 2, 17, and 23 days post inoculation. Data represent the mean of $n=6$; the same letters within a graph indicate no significant differences assessed by Fisher's LSD test $(P \leq 0.05)$ after performing ANOVA. 


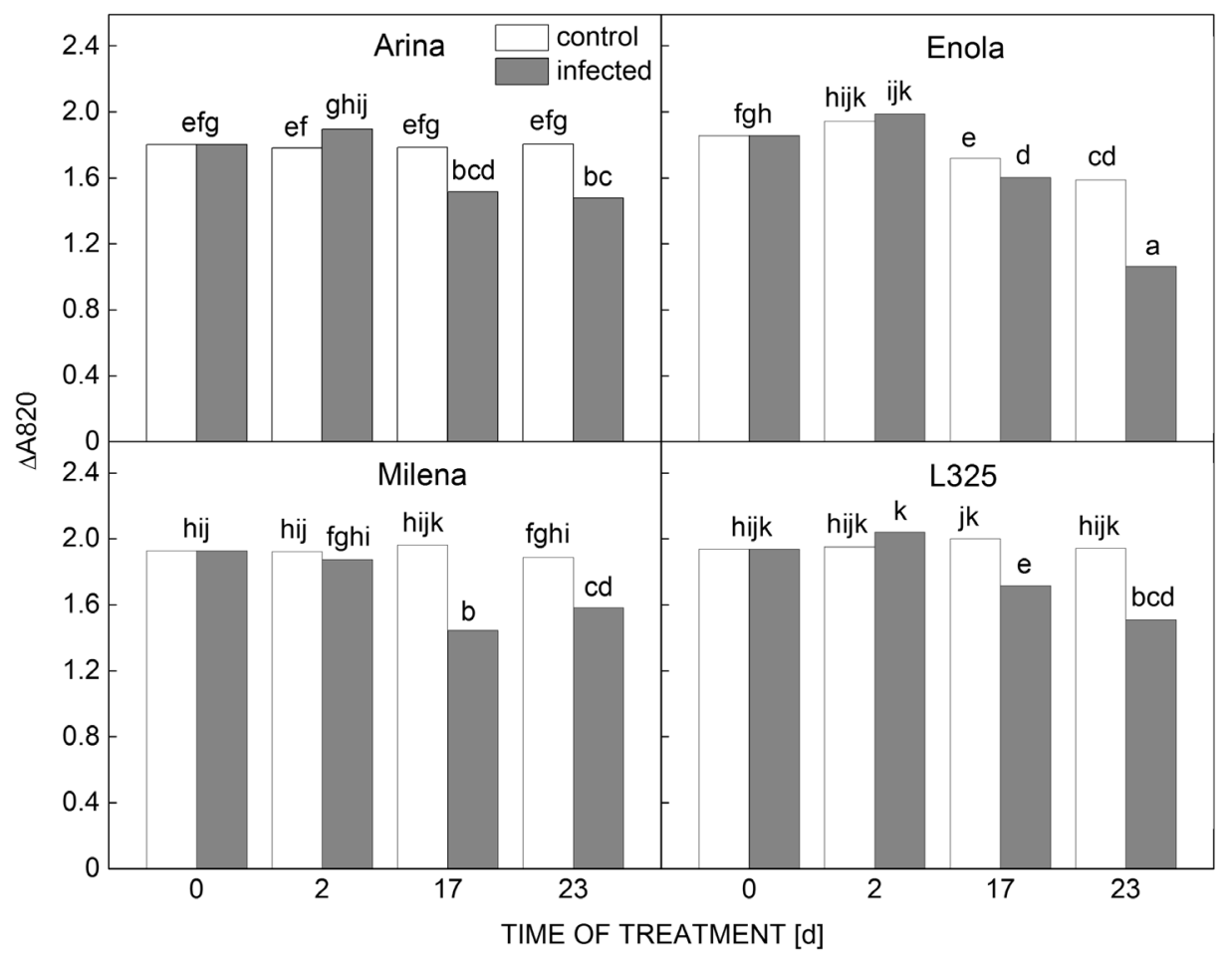

Fig. 6. Effect of inoculation of wheat genotypes Arina (highly resistant), Enola (highly susceptible), Milena, and L325 (moderately resistant) with Zymoseptoria tritici at 0,2, 17, and 23 days post inoculation on the photochemical activity of PSI, evaluated by leaf absorption changes at $\triangle \mathrm{A}_{820}$ after far-red illumination. Data represent the mean of $n=6$; the same letters within a graph indicate no significant differences assessed by Fisher's LSD test $(P \leq 0.05)$ after performing ANOVA.

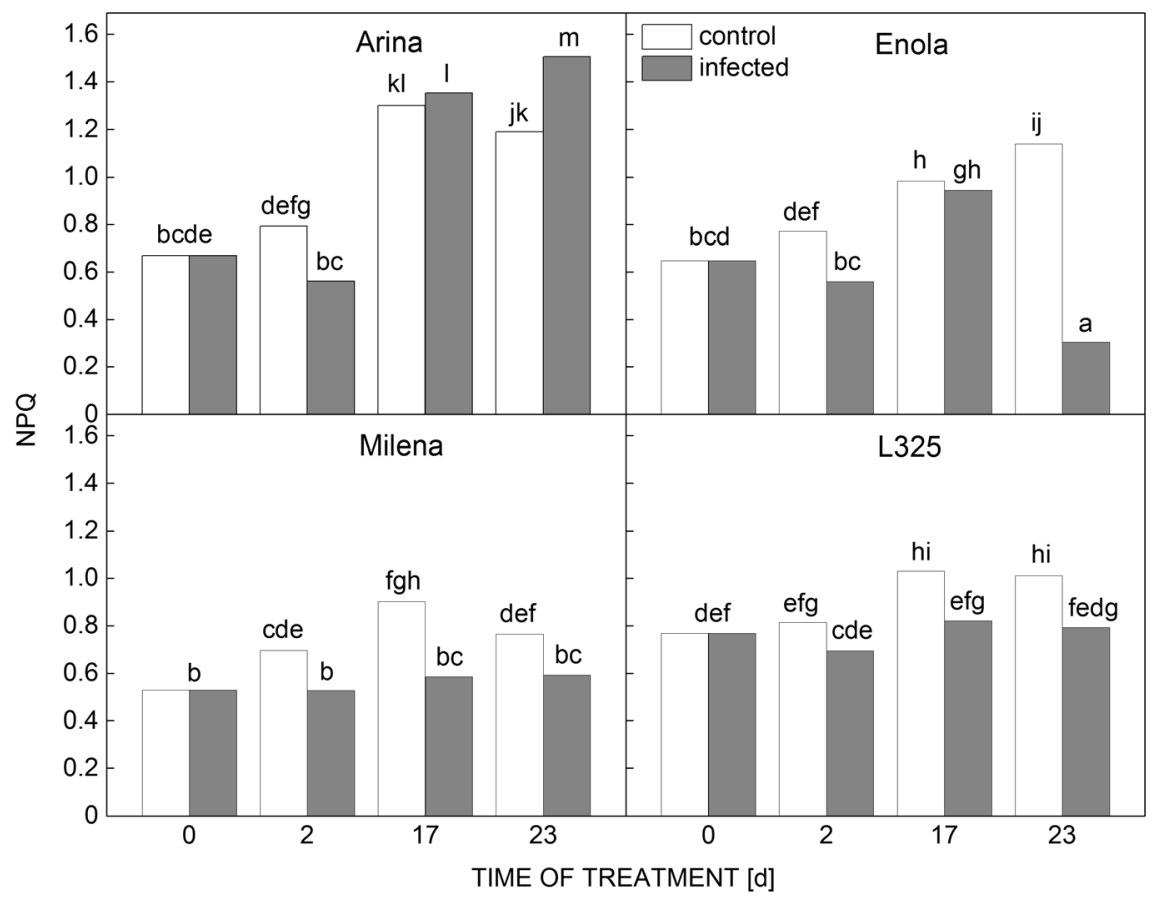

Fig. 7. Effect of inoculation with Zymoseptoria tritici on nonphotochemical quenching, NPQ, of wheat genotypes Arina (highly resistant), Enola (highly susceptible), Milena, and L325 (moderately resistant) measured at 0,2, 17, and 23 days post inoculation. Data

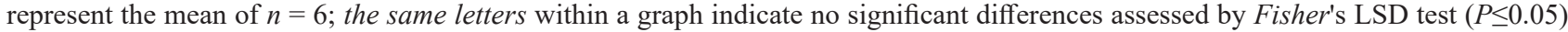
after performing $A N O V A$. 
of PSI $\left(\Delta \mathrm{A}_{820}\right)$ are presented in Fig. 6 . The values of $\Delta \mathrm{A}_{820}$ of control plants declined by 8 and $14 \%$ after 17 and $23 \mathrm{~d}$ in cv. Enola, whereas they did not change significantly in the other wheat genotypes. The PSI activity decreased as a result of fungal infection but it was lower than that of $\Phi_{\text {PSII }}$. Comparison of $\Delta \mathrm{A}_{820}$ results at $23 \mathrm{dpi}$ showed the similar reduction in PSI activity in cvs. Arina, Milena, and line L325 and the lowest PSI activity in cv. Enola.

Surprisingly, no significant changes in $g_{\text {s }}$ were detected in control and infected plants from cv. Arina, Milena, and line L325 (data not shown). At 23 dpi, an enormous enhancement in $g_{\mathrm{s}}$ was recorded in infected leaves of $\mathrm{cv}$. Enola, where small droplets of water were observed on and around the lesions with melanizing pycnidia (Fig. 5S).

The thermal energy dissipation, expressed as nonphotochemical quenching (NPQ), gradually increased in control plants up to $23 \mathrm{~d}$ (Fig. 7). However, statistically significant enhancement of NPQ at 23 dpi was observed only in cv. Arina, whereas the values of NPQ decreased by $20 \%$ in cv. Milena and line L325 and by $73 \%$ in cv. Enola. In contrast, the relative proportion of the energy absorbed and dissipated as heat in the PSII antennae, $1-\left(\mathrm{F}_{\mathrm{v}}{ }^{\prime} / \mathrm{F}_{\mathrm{m}}{ }^{\prime}\right)$, increased in all cultivars at $23 \mathrm{dpi}$, similarly in cvs. Arina, Milena, and line L325, and to the greatest extent in $\mathrm{cv}$. Enola (Fig. 6S).

\section{Discussion}

In the present study, we compared the effect of $Z$. tritici inoculation on some physiological parameters in four winter wheat genotypes varying in level of their resistance. The highly susceptible cv. Enola, presented the higher necrosis and pycnidia density than that of Arina, the highly resistant one (Stb15, Stb6, and partial resistance). In cv. Milena and line L325, the moderately resistant genotypes (unknown resistance factors), necrosis developed more than in Arina with none or a few pycnidia. Under noninfectious conditions, the plants of all genotypes remained healthy.

Across the time course of infection, RWC slightly decreased only at $23 \mathrm{dpi}$, indicating that the observed inhibition of the physiological activity of investigated genotypes was not related to the water content of leaves. The results clearly showed well expressed differences in the membrane stability of the genotypes especially at $23 \mathrm{dpi}$ and they were in line with their pathogen resistance. While the membrane integrity of the resistant genotype Arina was well protected after inoculation with $Z$. tritici, the most susceptible cv. Enola showed enormous enhancement in electrolyte leakage and the highest content of MDA at 23 dpi, suggesting membrane damage, a high level of lipid peroxidation, and oxidative stress as well. The changes in the content of MDA are a valuable indicator of a cellular damage caused by fungal infection (Dallagnol et al. 2011). It has been suggested that lipid peroxidation in cell membranes that results from oxidative stress promoted by pathogen infection can lead to the release of organelles and electrolytes (Mandal et al. 2008). In fact, high correlation coefficient of Pearson was determined for the changes in MDA and electrolyte leakage in infected leaves, $r=$ 0.991, for the most susceptible cultivar Enola. Thus, it could be proposed that plants of cv. Enola experienced oxidative stress as a result of fungal infection, which could be responsible for the enormous enhancement in electrolyte leakage and membrane damage. In addition, the strong enhancement in the MDA content and electrolyte leakage at 23 dpi in this cultivar was accompanied by the significantly increased proline content. In fact, the proline content was not affected by inoculation in the resistant $\mathrm{cv}$. Arina.

The significant reduction in Chl $(a+b)$ content was observed with development of the symptoms in infected plants, suggesting stimulated leaf senescence, which was different in the wheat genotypes studied. It has been reported that the decrease in the content could be, at least in part, due to the destruction of $\mathrm{Chl}$ and/or the deterioration of chloroplast membrane by the lipid peroxidation (Matsuyama 1983). Indeed, the high correlation coefficient of Pearson was determined for the changes in MDA and Chl content in infected leaves, especially in the most susceptible cv. Enola ( $r=-0.997)$.

Chl fluorescence has been widely used for assessing the resistance of plants to foliar pathogens (Bauriegel et al. 2011). Analysis of Chl fluorescence is noninvasive and therefore time courses can be performed on the same plant material. Our results showed that the photosynthetic activity was not significantly influenced in the presymptomatic period of infection by $Z$. tritici. Similar results have been obtained by other investigations on STB (Cornish et al. 1990, Robert et al. 2006) and also on bean anthracnose caused by Colletotrichum lindemuthianum (Bassanezi et al. 2001). In addition, the photochemical efficiency was not significantly affected in a chlorotic phase, which was consistent with previous studies showing that the chlorotic regions surrounding sporulating areas were still photosynthetically active for rust diseases (Scholes and Rolfe 1996). The effect of disease on leaf photosynthesis increased with the transition from chlorotic to necrotic symptoms and the most significant differences between the cultivars were observed at $23 \mathrm{dpi}$. The strongest reduction of quantum efficiency of both photosystems was observed in cv. Enola, but it should be mentioned that PSI was less inhibited compared to PSII. The decline in photochemical activity in the most susceptible cv. Enola was accompanied by an accumulation of closed PSII reaction centers, estimated by the changes in $1-\mathrm{q}_{\mathrm{p}}$, so called excitation pressure (data not shown). Considering the strong reduction of the $\mathrm{Chl}$ content in the infected leaves at $23 \mathrm{dpi}$, photosynthetic activity was not as strongly inhibited as could be expected. It was reported that some pathogens did not disturb photosynthetic activity in the remaining green area of infected leaves (van Oijen 1990), while the others even increased the photosynthetic rate of undamaged leaf sections, resulting in compensatory photosynthesis in the diseased plants (Berger et al. 2007). Using Chl fluorescence imaging, it was shown that the changes in photosynthesis upon infection were local and complexity and heterogeneity of effects were revealed (Berger et al. 2007).

Excitation pressure has been suggested to be a major prerequisite for the induction of efficient dissipation of the 
excess excitation energy thus protecting the PSII reaction center from overexcitation. Our results clearly showed that zeaxanthin-dependent nonphotochemical quenching, NPQ, had a protective effect at 23 dpi only in the resistant cv. Arina. The values of NPQ sharply decreased at $23 \mathrm{dpi}$ in the most sensitive cv. Enola. On the other hand, the reduction of photochemical activity in infected leaves was accompanied with increased proportion of energy dissipated as heat in the PSII antennae $\left(1-\mathrm{F}_{\mathrm{v}}{ }^{\prime} / \mathrm{F}_{\mathrm{m}}{ }^{\prime}\right.$, Demmig-Adams et al. 1996), which probably reflected the inability of the plants to regulate their mechanisms of photoprotection, thereby resulting in photooxidative damage to the infected tissue (Berger et al. 2007, Rios et al. 2017). Indeed, the highest enhancement in $1-\mathrm{F}_{\mathrm{v}}{ }^{\prime} / \mathrm{F}_{\mathrm{m}}{ }^{\prime}$ values was observed in susceptible cv. Enola at $23 \mathrm{dpi}$.

In summary, comparison of the effect of $Z$. tritici inoculation on physiological activity of winter wheat genotypes with different level of resistance showed that keeping the membrane integrity and the extent of oxidative stress is of significant importance for plant pathogen resistance. Analysis of the changes in different physiological and biochemical parameters in response to fungal infection showed that the measurements of electrolyte leakage, MDA content, and the chlorophyll fluorescence parameters, $\Phi_{\mathrm{PSII}}$ and NPQ, can be useful to screen the degree of tolerance of different wheat genotypes to Septoria tritici blotch.

\section{References}

Arraiano L.S., Chartrain L., Bossolini E. et al.: A gene in European wheat cultivars for resistance to an African isolate of Mycosphaerella graminicola. - Plant Pathol. 56: 73-78, 2007.

Bassanezi R.B., Amorim L., Filho A.B. et al.: Accounting for photosynthetic efficiency of bean leaves with rust, angular leaf spot, and anthracnose to assess crop damage. - Plant Pathol. 50: 443-452, 2001.

Bates L.S., Waldren S.P., Teare I.D.: Rapid determination of proline for water-stressed studies. - Plant Soil 39: 205-207, 1973.

Bauriegel E., Giebel A., Herppich W.B.: Hyperspectral and chlorophyll fluorescence imaging to analyse the impact of Fusarium culmorum on the photosynthetic integrity of infected wheat ears. - Sensors-Basel 11: 3765-3779, 2011.

Berger S., Sinha A.K., Roitsch T.: Plant physiology meets phytopathology: plant primary metabolism and plantpathogen interactions. - J. Exp. Bot. 58: 4019-4026, 2007.

Bilger W., Björkman O.: Role of the xanthophyll cycle in photoprotection elucidated by measurements of light-induced absorbance changes, fluorescence and photosynthesis in leaves of Hedera canariensis. - Photosynth. Res. 25: 173185, 1990.

Brown J.K.M., Kema G.H.J., Forrer H.-F. et al.: Resistance of wheat cultivars and breeding lines to Septoria tritici leaf blotch caused by isolates of Mycosphaerella graminicola in field trials. - Plant Pathol. 50: 325-338, 2001.

Brunner P.C., Torriani S.F.F., Croll D. et al.: Coevolution and life cycle specialization of plant cell wall degrading enzymes in a hemibiotrophic pathogen. - Mol. Biol. Evol. 30: 1337-1347, 2013.

Cornish P.S., Baker G.R., Murray G.M.: Physiological responses of wheat (Triticum aestivum) to infection with Mycosphaerella graminicola causing Septoria tritici blotch. - Aust. J. Agr.
Res. 41: 317-327, 1990.

Dallagnol L.J., Rodrigues F.A., Martins S.C.V. et al.: Alterations on rice leaf physiology during infection by Bipolaris oryzae. - Austral. Plant Path. 40: 360-365, 2011.

Dean R., Van Kan J.A., Pretorius Z.A. et al.: The top 10 fungal pathogens in molecular plant pathology. - Mol. Plant Pathol. 13: 414-430, 2012.

Demmig-Adams B., Adams III W.W., Barker D.H. et al.: Using chlorophyll fluorescence to assess the fraction of absorbed light allocated to thermal dissipation of excess excitation. Physiol. Plantarum 98: 253-264, 1996.

Esterbauer H., Cheeseman K.H.: Determination of aldehydic lipid peroxidation products: malonaldehyde and 4-hydroxynonenal. - Method. Enzymol. 186: 407-421, 1990.

Eyal Z., Scharen A.L., Prescott J.M. et al.: The Septoria Diseases of Wheat: Concepts and Methods of Disease Management. Pp. 52. CIMMYT, Mexico 1987.

Fones H., Gurr S.: The impact of Septoria tritici blotch disease on wheat: An EU perspective. - Fungal Genet. Biol. 79: 3-7, 2015.

Genty B., Briantais J.M., Baker N.R.: The relationship between the quantum yield of photosynthetic electron transport and quenching of chlorophyll fluorescence. - BBA-Gen. Subjects 990: 87-92, 1989.

Kema G.H.J., Yu D.Z., Rijkenberg F.H.J. et al.: Histology of the pathogenesis of Mycosphaerella graminicola in wheat. Phytopathology 86: 777-786, 1996.

van Kooten O., Snel J.: The use of chlorophyll fluorescence nomenclature in plant stress physiology. - Photosynth. Res. 25: $147-150,1990$.

Lee W.S., Rudd J.J., Hammond-Kosack K.E., Kanyuka K.: Mycosphaerella graminicola LysM effector-mediated stealth pathogenesis subverts recognition through both CERK1 and CEBiP homologues in wheat. - Mol. Plant Microbe In. 27: 236-243, 2014.

Lichtenthaler H.K.: Chlorophylls and carotenoids: pigments of photosynthetic biomembranes. - Method. Enzymol. 148: 350$382,1987$.

Lichtenthaler H.K., Babani F., Langsdorf G.: Chlorophyll fluorescence imaging of photosynthetic activity in sun and shade leaves of trees. - Photosynth. Res. 93: 235-244, 2007.

Mandal S., Mitra A., Mallick N.: Biochemical characterization of oxidative burst during interaction between Solanum lycopersicum and Fusarium oxysporum f. sp. lycopersici. Physiol. Mol. Plant P. 72: 56-61, 2008.

Matsuyama N.: Time-course alteration of lipid peroxidation and the activities of superoxide dismutase, catalase and peroxidase in blast-infected rice leaves. - Ann. Phytopathol. Soc. Japan 49: 270-273, 1983.

van Oijen M.: Photosynthesis is not impaired in healthy tissue of blighted potato plants. - Neth. J. Plant Pathol. 96: 55-63, 1990.

Orton E.S., Rudd J.J., Brown J.K.M.: Early molecular signatures of responses of wheat to Zymoseptoria tritici in compatible and incompatible interactions. - Plant Pathol. 66: 450-459, 2017.

Palma-Guerrero J., Torriani S.F., Zala M. et al.: Comparative transcriptomic analyses of Zymoseptoria tritici strains show complex lifestyle transitions and intraspecific variability in transcription profiles. - Mol. Plant Pathol. 17: 845-859, 2016.

Rios J.A., Aucique-Pérez C.E., Debona D. et al:: Changes in leaf gas exchange, chlorophyll $a$ fluorescence and antioxidant metabolism within wheat leaves infected by Bipolaris sorokiniana. - Ann. Appl. Biol. 170: 189-203, 2017.

Robert C., Bancal M.-O., Lannou C., Ney B.: Quantification of the effects of Septoria tritici blotch on wheat leaf gas 
exchange with respect to lesion age, leaf number, and leaf nitrogen status. - J. Exp. Bot. 57: 225-234, 2006.

Rosielle A.A.: Sources of resistance in wheat to speckled leaf blotch caused by Septoria tritici. - Euphytica 21: 152-161, 1972.

Rudd J.J., Kanyuka K., Hassani-Pak K. et al.: Transcriptome and metabolite profiling of the infection cycle of Zymoseptoria tritici on wheat reveals a biphasic interaction with plant immunity involving differential pathogen chromosomal contributions and a variation on the hemibiotrophic lifestyle definition. - Plant Physiol. 167: 1158-1185, 2015.

Scholes J.D., Rolfe S.A.: Photosynthesis in localised regions of oat leaves infected with crown rust (Puccinia coronata): quantitative imaging of chlorophyll fluorescence. - Planta 199: 573-582, 1996.

Schreiber U., Schliwa U., Bilger W.: Continuous recording of photochemical and non-photochemical chlorophyll fluorescence quenching with a new type of modulation fluorometer. - Photosynth. Res. 10: 51-62, 1986.

Steinberg G.: Cell biology of Zymoseptoria tritici: Pathogen cell organization and wheat infection. - Fungal Genet. Biol. 79: 17-23, 2015.

Zadoks J.C., Chang T.T., Konzak C.F.: A decimal code for growth stages of cereals. - Weed Res. 14: 415-421, 1974.

(C) The authors. This is an open access article distributed under the terms of the Creative Commons BY-NC-ND Licence. 